\title{
Phase space path integral in curved space
}

\author{
Rafael Ferraro ${ }^{1,2, *}$ and Mauricio Leston ${ }^{1, \dagger}$ \\ ${ }^{1}$ Instituto de Astronomía y Física del Espacio, \\ Casilla de Correo 67, Sucursal 28, 1428 Buenos Aires, Argentina \\ ${ }^{2}$ Departamento de Física, Facultad de Ciencias Exactas y Naturales, \\ Universidad de Buenos Aires, Ciudad Universitaria, Pabellón I, \\ 1428 Buenos Aires, Argentina
}

\begin{abstract}
Phase space path integral is worked out in a riemannian geometry, by employing a prescription for the infinitesimal propagator that takes riemannian normal coordinates and momenta on an equal footing. The operator ordering induced by this prescription leads to the DeWitt curvature coupling in the Schrödinger equation.
\end{abstract}

PACS 03.65.Ca

\footnotetext{
*ferraro@iafe.uba.ar

${ }^{\dagger}$ mauricio@iafe.uba.ar
} 


\section{Introduction}

The research on path integral in curved space began with DeWitt|1], who found that the measure of Pauli-Morete 2], adapted for curvilinear coordinates, leads to a coupling with the curvature in the Schrödinger equation (see also Ref.[3]). Later on Parker [4 shown that one can take profit of the ambiguous integration measure in the configuration space to generate different values for the coupling constant in the wave equation. This coupling can be understood as a contribution of an ambiguous measure, which can be written in an exponential way to dress it up as a potential $\xi \hbar^{2} R$ in the action. The ambiguity in the choice of the measure is nothing but the reflection of the different operator orderings for the Hamiltonian operator in curved space.

The ambiguities characterizing path integration methods in curved space, can be reexamined in the context of phase space variables. In phase space the integration measure seems to be better established: the Liouville measure $d q d p$ is privileged because it is invariant under canonical transformations. However, as was demonstrated by Kuchař [5], ambiguities are still present in phase space. Namely, the prescription for skeletonizing the canonical functional action fails in being clean, as it is in configuration space. So different prescriptions again lead to different curvature coupling in the wave equation (see also Ref. [6], and Ref. [7] for the handling of a relativistic system). In order to fix the ambiguities, it has been proposed [8] that the natural skeletonization of the canonical functional action should be one managing canonical coordinates and momenta on an equal footing. The skeletonized canonical functional action proposed in Ref. [8] is based on those complete solutions of the Hamilton-Jacobi equation that accomplish boundary conditions which are symmetric in coordinates and momenta. The aim of the present paper is to apply the results of Ref. [8] to a phase space path integral in curved space, and compare the result with DeWitt and Parker prescriptions for configuration space path integration. In Section 2 we review Parker's path integral in configuration space. In Section 3 we present the phase space path integral proposed in Ref.[8]. In Section 4 we apply this later prescription to the system studied by DeWitt and Parker. In Section 5 we display the conclusions.

\section{Configuration space path integral}

The propagator $K\left(q^{\prime} t^{\prime} \mid q t\right)$ is a two point function that builds those solution $\Psi\left(q^{\prime}, t^{\prime}\right)$ of the Schrödinger equation satisfying a given initial condition $\Psi_{o}(q)$ at time $t$ :

$$
\Psi\left(q^{\prime}, t^{\prime}\right)=\int d q K\left(q^{\prime} t^{\prime} \mid q t\right) \Psi_{o}(q)
$$

Thus the propagator itself must be a solution of the Schrödinger equation, accomplishing the boundary condition

$$
\lim _{t^{\prime} \rightarrow t^{+}} K\left(q^{\prime} t^{\prime} \mid q t\right)=\delta\left(q^{\prime}, q\right),
$$


and the composition rule

$$
K\left(q^{\prime \prime} t^{\prime \prime} \mid q^{\prime} t^{\prime}\right)=\int K\left(q^{\prime \prime} t^{\prime \prime} \mid q t\right) d q K\left(q t \mid q^{\prime} t^{\prime}\right) .
$$

When curvilinear coordinates are used, one should care about the geometrical character of the quantities involved in Eq.(1D). In fact, the inner product

$$
\left\langle\Psi_{1}, \Psi_{2}\right\rangle=\int d q \Psi_{1}^{*}(q) \Psi_{2}(q)
$$

must be invariant under general coordinate changes. So the wave functions must be regarded as densities of weight $1 / 2$, in order that $d q \Psi_{1}^{*}(q) \Psi_{2}(q)$ results to be an invariant volume. Some people prefer regarding the wave functions as invariant; then they add a factor $\sqrt{g}$ in the inner product ( $g$ is the determinant of the metric). The latter choice amounts the replacement $\Psi \rightarrow g^{1 / 4} \Psi$, and the consequent redefinition of the operators. In this paper we will attach ourselves to the former choice. Thus, we will regard the propagator as a bidensity of weight $1 / 2$.

The propagator can be formally expressed as a path integral [9]

$$
K\left(q^{\prime} t^{\prime} \mid q t\right)=\int \mathcal{D} q(t) \exp \left[\frac{i}{\hbar} S[q(t)]\right]
$$

where the integration is performed on all paths joining the arguments of the propagator. This functional integration can be dismantled into infinitesimal (short time) propagators $K_{\varepsilon}\left(q^{\prime}, q\right) \equiv K\left(q^{\prime} t+\varepsilon \mid q t\right)$, which can be assembled by means of the composition rule (3), so rebuilding the finite time propagator. Each infinitesimal piece must be a bidensity of weight $1 / 2$. This skeletonization process give sense to the otherwise ill-defined functional integration (5). However the choice for the infinitesimal pieces is not unique. Thus path integration is an ambiguous operation: each choice for the skeletonization amounts a different quantization of the system, all of them possessing the same classical limit.

The infinitesimal propagator $K_{\varepsilon}\left(q^{\prime}, q\right)$ is defined by developing the time evolution of the wave function in the following way:

$$
\Psi\left(q^{\prime}, t\right)+\varepsilon \frac{\partial}{\partial t} \Psi\left(q^{\prime}, t\right)=\int d^{n} q K_{\varepsilon}\left(q^{\prime}, q\right) \Psi(q, t)+O\left(\varepsilon^{2}\right) .
$$

One can recover the wave equation in Eq.(6) by retaining only the terms that are linear in $\varepsilon$. For this aim one replaces $\Psi(q, t)$ in the integrand of the Eq.(可) by the expansion

$$
\Psi(q, t)=\Psi\left(q^{\prime}, t\right)+\frac{\partial \Psi}{\partial q^{\prime i}}\left(q^{\prime}, t\right) \xi^{i}+\frac{1}{2} \frac{\partial \Psi}{\partial q^{\prime i} \partial q^{\prime j}}\left(q^{\prime}, t\right) \xi^{i} \xi^{j}+O\left(\xi^{3}\right)
$$


where $\xi^{i}=q^{i}-q^{\prime i}$.

If the system io governed by the Lagrangian

$$
L=\frac{1}{2} g_{i j}(q) \dot{q}^{i} \dot{q}^{j}, \quad i=1, \ldots, n .
$$

then the Schrödinger equation io a second order invariant equation in curved space, whose general form is:

$$
i h \frac{\partial}{\partial t} \Psi(q, t)=-\frac{1}{2} \hbar^{2} \triangle^{(1 / 2)} \Psi(q, t)+\mu \hbar^{2} R \Psi(q, t)
$$

where $\triangle^{(1 / 2)}$ is the Laplacian for densities of weight $1 / 2: \triangle^{(1 / 2)}=g^{1 / 4} \triangle g^{-1 / 4}$, $R$ is the curvature escalar of the manifold and $\mu$ is a real number. Therefore, the terms $O\left(\xi^{3}\right)$ in Eq. (7) cannot generate contributions linear in $\varepsilon$ when they are replaced in Eq. (6). In Eq. (9), the coupling constant $\mu$ is specified by giving a prescription to path integrate the propagator (5) or, equivalently, to order the hamiltonian operator.

Parker's infinitesimal propagator for the system (8) can be expressed in the following way [4:

$$
\begin{gathered}
K_{\varepsilon}\left(q^{\prime} \mid q\right)=\varepsilon^{n(p-1 / 2)} \frac{g\left(q^{\prime}\right)^{1 / 4} g(q)^{1 / 4}}{(2 \pi i \hbar)^{n / 2}}\left(g\left(q^{\prime}\right)^{-1 / 2} D_{\varepsilon}\left(q^{\prime}, \mid q\right) g(q)^{-1 / 2}\right)^{p} \\
\exp \left[\frac{i}{\hbar} S_{\varepsilon}\left(q^{\prime}, \mid q\right)\right]
\end{gathered}
$$

where $D\left(q^{\prime} t^{\prime} \mid q t\right)$ is the Van Vleck determinant 10

$$
D\left(q^{\prime} t^{\prime} \mid q t\right) \equiv \operatorname{det}\left(-\frac{\partial^{2} S\left(q^{\prime} t^{\prime}, q t\right)}{\partial q^{\prime} \partial q}\right)
$$

and $S\left(q^{\prime} t^{\prime} \mid q t\right)$ is the Hamilton principal function, i.e. the functional action $S=\int L d t$ evaluated on its stationary (classical) path.

Parker's propagator (10) satisfies the wave equation

$$
i h \frac{\partial}{\partial t^{\prime}} K\left(q^{\prime} t^{\prime} \mid q t\right)=\left[-\frac{1}{2} \hbar^{2} \Delta^{\prime(1 / 2)}+\frac{1}{6}(1-p) \hbar^{2} R\left(q^{\prime}\right)\right] K\left(q^{\prime} t^{\prime} \mid q t\right) .
$$

The choice $p=1 / 2$ in Eq. (10) is the DeWitt's propagator [1], while $p=0$ corresponds to the Feynman's propagator 11].

By composing the infinitesimal pieces $(10)$ one gets a meaning for the path integral (5). Thus the Van Vleck determinants take part in the measure $\mathcal{D} \mathbf{q}(t)$ and the Hamilton principal functions $S\left(q^{\prime} t^{\prime} \mid q t\right)$ are the pieces that skeletonize the functional action $S[q(t)]$ : 


$$
S[q(t)] \longrightarrow \sum_{k=0}^{N-1} S\left(q_{k+1} t_{k+1} \mid q_{k} t_{k}\right)
$$

where $t_{k+1}-t_{k}=\varepsilon$, and $q_{k}$ is a shorthand for $q^{i}\left(t_{k}\right)$.

\section{Phase space path integral}

Phase space path integral,

$$
K\left(q^{\prime} t^{\prime} \mid q t\right)=\int \mathcal{D} p(t) \mathcal{D} q(t) \exp \left[\frac{i}{\hbar} S[q(t), p(t)]\right],
$$

also requires a skeletonized version to make sense. The infinitesimal propagator proposed in Ref. [8] is

$$
\begin{gathered}
K_{\varepsilon}\left(q^{\prime}, q\right)=K\left(q^{\prime \prime} t^{\prime \prime}=t^{\prime}+\varepsilon \mid q^{\prime} t^{\prime}\right)=\int \frac{d^{n} p_{a}}{(2 \pi \hbar)^{n}}\left|\operatorname{det} \frac{\partial^{2} J\left(q^{\prime \prime} t^{\prime \prime} \mid p t\right)}{\partial q^{\prime \prime} j p_{a}}\right|^{1 / 2} \\
\left|\operatorname{det}-\frac{\partial^{2} J\left(p t \mid q^{\prime} t^{\prime}\right)}{\partial p_{a} \partial q^{\prime}}\right|^{1 / 2} \exp \left[\frac{i}{\hbar}\left(J\left(q^{\prime \prime} t^{\prime \prime} \mid p t\right)+J\left(p t \mid q^{\prime} t^{\prime}\right)\right)\right]
\end{gathered}
$$

Here $J\left(q t^{\prime} \mid p t\right)$ and $J\left(p t \mid q t^{\prime}\right)$ are two invariant functions related to the dynamical system, which should take on an equal footing canonical coordinates and momenta. Besides these functions should provide a skeletonized version of the canonical functional action $S[q(t), p(t)]$ :

$$
S[q(t), p(t)] \longrightarrow \sum_{k=0}^{(N-2) / 2}\left\{J\left(q_{2 k+2} t_{2 k+2} \mid p_{2 k+1} t_{2 k+1}\right)+J\left(p_{2 k+1} t_{2 k+1} \mid q_{2 k} t_{2 k}\right)\right\}
$$

The functions $J\left(q t^{\prime} \mid p t\right)$ and $J\left(p t \mid q t^{\prime}\right)$ used in Ref. [8] are two complete solutions of the Hamilton-Jacobi equation linked by the relation

$$
J\left(q t^{\prime} \mid p t\right)=-J\left(p t \mid q t^{\prime}\right)
$$

The invariance of $J\left(q t^{\prime} \mid p t\right)$ is not guaranteed by the invariant Hamiltonian in the Hamilton-Jacobi equation, because the boundary condition must also be invariant. The boundary condition proposed in Ref. [8] is

$$
J\left(q^{i} t \mid p_{a} t\right)=p_{a} \sigma^{a}\left(q^{j}\right),
$$

$\sigma^{a}\left(q^{j}\right)$ in Eq.(18) are riemannian normal coordinates for the point $P$ identified by the coordinates $q^{i}$, based at some origin $O$ :

$$
\sigma^{a}\left(q^{j}\right)=s(O, P) u^{a},
$$


where $s(O, P)=\int_{O}^{P} \sqrt{g_{i j} d q^{i} d q^{j}}$ is the (invariant) lenght of the geodesic joining $O$ and $P$, and $u^{a}$ are components of the unitary vector at $O$ that is tangent to the geodesic joining $O$ and $P$, in some basis chosen at $O$. Riemannian normal coordinates have a twofold behavior: like $s$ they are invariant under changes of coordinates $q^{i}$, but transform as components of a vector under changes of basis in the target space $T_{O} \cdot p_{a}$ in Eqs.(15) and (18) are momenta canonically conjugated to $\sigma^{a}\left(q^{j}\right)$,

$$
p_{j}=\frac{\partial \sigma^{a}}{\partial q^{j}}\left(q^{i}\right) p_{a}
$$

Thus the boundary condition (18) is invariant under both general changes of the $q^{i}$ 's and change of basis in the target space $T_{O}$. The boundary condition (18) means that canonically conjugated riemannian normal coordinates and momenta enter the skeletonization on an equal footing.

As is shown in Ref. $\mid 8 \|, J\left(q^{\prime} t^{\prime} \mid p t\right)$ and $J\left(p^{\prime} t^{\prime} \mid q t\right)$, there named Jacobi principal functions, result to be the Legendre transforms of $S\left(\sigma^{a}\left(q^{\prime}\right) t^{\prime} \mid \sigma^{a}(q) t\right)$ that interchange the variables $\sigma^{a}$ and $p_{a}$ at $t$ and $t^{\prime}$ respectively. Then $J\left(q^{\prime} t^{\prime} \mid p t\right)$ generates contact transformations

$$
p_{j}^{\prime}=\frac{\partial J\left(q^{\prime i} t^{\prime} \mid p_{a} t\right)}{\partial q^{\prime j}}, \quad \sigma^{a}=\frac{\partial J\left(q^{\prime i} t^{\prime} \mid p_{a} t\right)}{\partial p_{a}} .
$$

So the determinants in the infinitesimal propagator (15) are well defined in all phase space. The functions $J\left(q t^{\prime} \mid p t\right)$ and $J\left(p t^{\prime} \mid q t\right)$ also provides a proper skeletonization for the canonical action:

$$
\begin{gathered}
J\left(q_{2 k+2} t_{2 k+2} \mid p_{2 k+1} t_{2 k+1}\right)+J\left(p_{2 k+1} t_{2 k+1} \mid q_{2 k} t_{2 k}\right) \\
\longrightarrow p_{a 2 k+1}\left(\sigma_{2 k+2}^{a}-\sigma_{2 k}^{a}\right)-H\left(\sigma_{2 k}^{a}, p_{j 2 k+1}, t_{2 k+1}\right)\left(t_{2 k+2}-t_{2 k}\right) .
\end{gathered}
$$

Differing from the infinitesimal propagator (10), which only holds for systems whose Lagrangian is quadratic in the velocities [12], the infinitesimal propagator (15) can be applied to any hamiltonian system. It will provide a unitary evolution when $t$ in Eq. (15) is the mid time $t \equiv t^{\prime}+(\varepsilon / 2)=t^{\prime \prime}-(\varepsilon / 2)$.

Finally we remark that it is by no means evident that the prescription (15) does not depend on the choice of the origin $O$ for defining normal riemannian coordinates. This topic will be analyzed in the next Section.

\section{Infinitesimal propagator in the riemannian geometry}

Let be $g_{a b}(q)$ the components of the metric tensor at $P$ in the riemannian normal coordinates system (RNCS),

$$
g_{a b}(q) \equiv \frac{\partial q^{j}}{\partial \sigma^{a}}(q) \frac{\partial q^{i}}{\partial \sigma^{b}}(q) g_{i j}(q) \quad g^{a b}(q) \equiv \frac{\partial \sigma^{a}}{\partial q^{i}}(q) \frac{\partial \sigma^{b}}{\partial q^{j}}(q) g^{i j}(q)
$$


$g_{a b}$ is invariant under changes of $q^{i}$ s, but is a tensor in $T_{O}$. Since $u^{a}$ in Eq.(19) is unitary $\left(g_{a b} u^{a} u^{b}=1\right)$, one recognizes that

$$
g_{a b} \sigma^{a} \sigma^{b}=s^{2} .
$$

Then,

$$
\sigma^{a}(q)=\frac{1}{2} g^{a b}(q) \frac{\partial s^{2}}{\partial \sigma^{b}}(q)=s g^{a b}(q) \frac{\partial s}{\partial \sigma^{b}}(q)
$$

and

$$
u^{a}(q)=g^{a b}(q) \frac{\partial s}{\partial \sigma^{b}}(q) .
$$

At the lowest order in $\varepsilon=\Delta t$, the solution of the Hamilton-Jacobi equation fulfilling the boundary condition (18) is

$$
\begin{gathered}
J_{\varepsilon}\left(q^{i} \mid p_{a}\right)=p_{a} \sigma^{b}\left(q^{i}\right)-\frac{1}{2} g^{a b} p_{a} p_{b} \varepsilon+O\left(\varepsilon^{2}\right) \\
\left|\frac{\partial^{2} J_{(\varepsilon / 2)}\left(q^{i}, p_{a}, \varepsilon\right)}{\partial q^{i} \partial p_{a}}\right|^{1 / 2}=\left|\frac{\partial \sigma^{a}}{\partial q^{i}}\right|^{1 / 2}\left(1-\frac{1}{4} \frac{\partial g^{a b}}{\partial \sigma^{a}} p_{b} \varepsilon\right)+O\left(\varepsilon^{2}\right)
\end{gathered}
$$

Both Jacobi principal functions, the one of Eq.(27) and its companion of Eq.(17), enter the Eq.(15) to compute the infinitesimal propagator. The result is (see Appendix)

$$
\begin{gathered}
K_{\varepsilon}\left(q^{\prime \prime} t^{\prime \prime}=t^{\prime}+\varepsilon \mid q^{\prime} t^{\prime}\right)=\frac{\varepsilon^{n / 2}}{(2 \pi i \hbar)^{n / 2}}\left[\operatorname{det} g_{i j}\left(q^{\prime \prime}\right)\right]^{-1 / 4} D_{\varepsilon}\left(q^{\prime \prime} \mid q^{\prime}\right)\left[\operatorname{det} g_{i j}\left(q^{\prime}\right)\right]^{-1 / 4} \\
\exp \frac{i}{\hbar}\left[S_{\epsilon}\left(q^{\prime \prime} \mid q^{\prime}\right)-\frac{\hbar^{2}}{2} \int_{t^{\prime}}^{t^{\prime \prime}} d t\left(\operatorname{det} g_{a b}\right)^{1 / 4} \triangle\left[\left(\operatorname{det} g_{a b}\right)^{-1 / 4}\right]\right]
\end{gathered}
$$

where the integral on $t$ is evaluated along the classical trajectory $q(t)$ joining $\left(q^{\prime}, t^{\prime}\right)$ and $\left(q^{\prime \prime}, t^{\prime \prime}\right)$. This infinitesimal propagator leads to the following wave equation:

$$
i h \frac{\partial}{\partial t} \Psi(q, t)=-\frac{\hbar^{2}}{2} \triangle^{(1 / 2)} \Psi(q, t)+\frac{1}{2} \hbar^{2}\left(\operatorname{det} g_{a b}\right)^{\frac{1}{4}} \triangle\left[\left(\operatorname{det}\left(g_{a b}\right)\right)^{-\frac{1}{4}}\right] \Psi(q, t) .
$$

The term $M_{O}(q) \equiv \frac{\hbar^{2}}{2}\left(\operatorname{det} g_{a b}(q)\right)^{\frac{1}{4}} \triangle\left[\left(\operatorname{det} g_{a b}(q)\right)^{-\frac{1}{4}}\right]$ is invariant under changes of the coordinates of point $P$, where the laplacian operates, and also under change of basis in $T_{O}$. Except for the factor $\exp \left[-\frac{i}{\hbar} \int_{t^{\prime}}^{t^{\prime \prime}} d t M_{O}(q(t))\right]$, the result (29) agrees with (10) for $p=1$. However this factor depends on the 
choice of the origin $O$, which is a non admissible dependence for the propagator. To be convinced that $M_{O}$ really depends on $O$, one can evaluate $M_{O}$ in RNCS:

$$
\begin{gathered}
\left(\operatorname{det} g_{a b}\right)^{1 / 4} \triangle\left(\operatorname{det} g_{a b}\right)^{-1 / 4}= \\
\left(\operatorname{det} g_{a b}\right)^{-1 / 4} \frac{\partial}{\partial \sigma^{a}}\left(\operatorname{det} g_{a b}\right)^{1 / 2} g^{a b} \frac{\partial\left(\operatorname{det} g_{a b}\right)^{-1 / 4}}{\partial \sigma^{b}}= \\
-\frac{1}{4} g^{a b} g^{c d} \frac{\partial^{2} g_{c d}}{\partial \sigma^{a} \partial \sigma^{b}}-\frac{1}{16} g^{c d} \frac{\partial g_{c d}}{\partial \sigma^{a}} g^{a b} g^{e f} \frac{\partial g_{e f}}{\partial \sigma^{b}}-\frac{1}{4} \frac{\partial g^{a b}}{\partial \sigma^{a}} g^{c d} \frac{\partial g_{c d}}{\partial \sigma^{b}}
\end{gathered}
$$

This expression can be compared with the curvature scalar $R(q)$ :

$$
\begin{gathered}
R=\frac{1}{2} \frac{\partial g_{a b}}{\partial \sigma^{c}} \frac{\partial g_{d e}}{\partial \sigma^{f}}\left(g^{a c} g^{d e} g^{b f}-g^{a b} g^{d e} g^{c f}+2 g^{d c} g^{b e} g^{a f}+2 g^{d f} g^{b e} g^{a c}\right) \\
+\frac{\partial^{2} g_{a b}}{\partial \sigma^{c} \partial \sigma^{d}}\left(g^{a c} g^{b d}-g^{a b} g^{c d}\right)
\end{gathered}
$$

Since they do not coincide, the term $M_{O}$ depends on the choice of the origin $O$ to define the $p_{a}$ 's ( in flat space $M_{o}$ is null because RNCS is the cartesian system where the derivatives of the metric are null) .

\section{Prescription for the origin of RNCS in the infinitesimal propagator}

In order to avoid any undesirable dependence on geometric objects non linked to the physical system, the point $O$ in (29) should be dictated by the dynamical system itself. So the point $O$ for the infinitesimal propagator (29) must be chosen on the classical path joining $q^{\prime \prime}\left(t^{\prime \prime}\right)$ and $q^{\prime}\left(t^{\prime}\right)$. This prescription will give a proper meaning to the factor $\exp \left[-\frac{i}{\hbar} \int_{t^{\prime}}^{t^{\prime \prime}} d t M_{O}(q(t))\right]$ in Eq. (29). The simplest prescription is to choose the point $O$ as the final point in the infinitesimal propagator. So $M_{O}(q)$ in Eq. (30) must be replaced by $M_{P}(q)$, being $P$ the point where the wave function is evaluated. Having into account the expansion $([5])$

$$
\operatorname{det}\left(\frac{\partial \sigma^{a}}{\partial q^{j}}\right)=g^{-\frac{1}{2}}(P) g^{-\frac{1}{2}}(O)\left(1+\frac{1}{6} s^{2} R^{i j} s_{, i} s_{, j}+O\left(s^{6}\right)\right),
$$

then

$$
\lim _{O \rightarrow P} M_{O}(q)=\frac{\hbar^{2}}{12} R(q)
$$

Thus the Schrödinger equation(30)turns out to be:

$$
i h \frac{\partial}{\partial t} \Psi(q, t)=-\frac{1}{2} \hbar^{2} \triangle^{(1 / 2)} \Psi(q, t)+\frac{1}{12} \hbar^{2} R \Psi(q, t)
$$


which exhibits the curvature coupling prescribed by DeWitt.

\section{Conclusions}

To some extent the prescription $O \equiv P$ in the infinitesimal propagator seems arbitrary, in the sense that any other point of the classical trajectory joining $q^{\prime}$ and $q^{\prime \prime}$ is equally natural to play that role. However, the result of Section 5 -the DeWitt curvature coupling- is not affected by a displacement of point $O$ along the classical path. In fact, such a displacement would change $M_{o}(q(t))$ to $M_{o}(q(t))+O\left(\xi^{a}\right)$; thus $K_{\varepsilon}\left(q^{\prime \prime} \mid q^{\prime}\right)$ would get a contribution that can be expanded in powers of $\xi^{a}$. Because of the rule $\xi^{a} \xi^{b} \longrightarrow i \varepsilon \hbar g^{a b}$ (see Eq. (39) in the Appendix), these changes do not modify the leading term in Eq. (6) but only contribute to higher order in $\varepsilon$. Then, these changes can be ignored at the level of the infinitesimal propagator. Therefore the DeWitt curvature coupling appears to be the natural one for a quadratic system.

\section{Appendix}

\section{Identities}

$$
\begin{gathered}
g_{i j, k}=-g_{i h} g^{h l},_{k} g_{l j} \\
g^{N},_{i}=N g^{N} g^{m n} g_{n m, i} \\
\int_{-\infty}^{\infty} \frac{d^{n} x}{(2 \pi)^{n / 2}} \exp \left[-\frac{1}{2}(x, A x)+(b, x)\right]=(\operatorname{det} A)^{-1 / 2} \exp \frac{1}{2}\left(b, A^{-1} b\right)
\end{gathered}
$$

where $A$ is a $n \times n$ matrix and $b$ and $x$ are vectors.

$$
\begin{gathered}
\int_{-\infty}^{\infty} \triangle q^{\alpha_{1}} \triangle q^{\alpha_{2}} \ldots \triangle q^{\alpha_{2 m}} \exp \left(\frac{i}{2 \hbar \varepsilon} g_{i j} \triangle q_{i} \triangle q_{j}\right) d^{n} q=(2 \pi \hbar \varepsilon)^{N / 2} g^{-1 / 2}(i \hbar \varepsilon)^{m} \\
\left\{g^{\alpha_{1} \alpha_{2}} \ldots g^{\alpha_{2 m-1} \alpha_{2 m}}\right\}+\text { all relevant permutations of } \alpha_{1}, \ldots, \alpha_{2 m}
\end{gathered}
$$

\section{Derivation of Wave Equation}


The expression (15) can be integrated with the Jacobi principal function (27). The result is:

$$
\begin{gathered}
K_{\varepsilon}\left(q^{\prime \prime} \mid q^{\prime}\right)=\frac{1}{(2 \pi \hbar \varepsilon)^{n / 2}} g^{\prime \frac{1}{4}} g^{\prime \prime \frac{1}{4}}\left(\operatorname{det}\left(g_{a b}^{\prime \prime}\right)\right)^{-\frac{1}{4}}\left(\operatorname{det}\left(g_{a b}^{\prime}\right)\right)^{-\frac{1}{4}}\left(\operatorname{det}\left(\bar{g}_{a b}\right)\right)^{1 / 2} \\
{\left[1+\frac{1}{4} \bar{g}_{a b}\left(\frac{\partial g^{\prime b d}}{\partial \sigma^{\prime d}}-\frac{\partial g^{\prime \prime b d}}{\partial \sigma^{\prime \prime b}}\right) \xi^{a}\right] \exp \left(\frac{i}{2 \hbar \varepsilon} \bar{g}_{a b} \xi^{a} \xi^{b}\right)}
\end{gathered}
$$

where $\bar{g}^{a b} \equiv \frac{1}{2}\left(g^{\prime \prime a b}+g^{\prime a b}\right)$. Let be $\xi^{j} \equiv \sigma^{\prime \prime j}-\sigma^{\prime j}$. Then:

$$
\begin{gathered}
\left.\bar{g}_{a b}\right|_{\xi=0}=g_{a b} \\
\left.\frac{\partial \bar{g}_{a b}}{\partial \sigma^{\prime c}}\right|_{\xi=0}=\frac{1}{2} \frac{\partial g_{a b}^{\prime}}{\partial \sigma^{\prime c}} \\
\left.\frac{\partial \bar{g}^{a b}}{\partial \sigma^{\prime c}}\right|_{\xi=0}=\frac{1}{2} \frac{\partial g^{\prime a b}}{\partial \sigma^{\prime c}} \\
\left.\frac{\partial^{2} \bar{g}_{a b}}{\partial \sigma^{\prime c} \partial \sigma^{\prime d}}\right|_{\xi=0}=\frac{1}{2} \frac{\partial^{2} g_{a b}^{\prime \prime}}{\partial \sigma^{\prime \prime c} \partial \sigma^{\prime \prime d}}+\frac{1}{4}\left(\frac{\partial g_{a m}^{\prime \prime}}{\partial \sigma^{\prime \prime c}} \frac{\partial g^{\prime \prime m n}}{\partial \sigma^{\prime \prime d}} g_{n b}^{\prime \prime}+g_{a m} \frac{\partial g^{\prime \prime m n}}{\partial \sigma^{\prime \prime d}} \frac{\partial g_{n b}^{\prime \prime}}{\partial \sigma^{\prime \prime c}}\right)
\end{gathered}
$$

By expanding $\operatorname{det}\left(g_{a b}^{\prime}\right)$ and $\operatorname{det}\left(\bar{g}_{a b}\right)$ around $\sigma^{\prime \prime a}$,

$\left(\operatorname{det} g_{a b}^{\prime}\right)^{-\frac{1}{4}}=\left(\operatorname{det} g_{a b}^{\prime \prime}\right)^{-\frac{1}{4}}-\frac{\partial\left(\operatorname{det}\left(g_{a b}^{\prime \prime}\right)\right)^{-\frac{1}{4}}}{\partial \sigma^{\prime \prime c}} \xi^{i}+\frac{1}{2} \frac{\partial^{2}\left(\operatorname{det}\left(g_{a b}^{\prime \prime}\right)\right)^{-\frac{1}{4}}}{\partial \sigma^{\prime \prime c} \partial \sigma^{\prime \prime}} \xi^{c} \xi^{d}+O\left(\xi^{3}\right)$

and using the identities (42-44), one obtains the terms that are relevant in the integration of Eq. (6). According to Eq.(39) these terms are:

$$
\begin{gathered}
\left(\operatorname{det} \bar{g}_{a b}\right)^{\frac{1}{2}}=\left(\operatorname{det} g_{a b}^{\prime \prime}\right)^{\frac{1}{4}}\left(\operatorname{det} g_{a b}^{\prime}\right)^{\frac{1}{4}}+\left(\operatorname{det} g_{a b}^{\prime \prime}\right)^{\frac{1}{2}} \frac{1}{16} \frac{\partial g^{\prime \prime a m}}{\partial \sigma^{\prime \prime d}} \frac{\partial g_{a m}^{\prime \prime}}{\partial \sigma^{\prime \prime c}} \xi^{c} \xi^{d}+O\left(\xi^{3}\right) \\
\bar{g}_{a b} \xi^{a}\left(\frac{\partial g^{\prime b c}}{\partial \sigma^{\prime c}}-\frac{\partial g^{\prime \prime b c}}{\partial \sigma^{\prime c}}\right)=\frac{\partial^{2} g_{a n}^{\prime \prime}}{\partial \sigma^{\prime \prime c} \partial \sigma^{\prime \prime d}} g^{\prime \prime n c} \xi^{a} \xi^{d}+ \\
g_{a b}^{\prime \prime} \frac{\partial g^{\prime \prime b m}}{\partial \sigma^{\prime \prime d}} \frac{\partial g_{m n}^{\prime \prime}}{\partial \sigma^{\prime \prime c}} g^{\prime \prime n c} \xi^{a} \xi^{d}+\frac{\partial g^{\prime \prime n c}}{\partial \sigma^{\prime \prime d}} \frac{\partial g_{a n}^{\prime \prime}}{\partial \sigma^{\prime \prime c}} \xi^{a} \xi^{d}+O\left(\xi^{3}\right) \\
\exp \frac{i}{2 \hbar \varepsilon} \bar{g}_{a b} \xi^{a} \xi^{b}=\exp \left[\frac{i}{2 \hbar \varepsilon} g_{a b} \xi^{a} \xi^{b}\right]\left\{1-\frac{i}{4 \hbar \varepsilon} \frac{\partial g_{a b}^{\prime \prime}}{\partial \sigma^{\prime \prime c}} \xi^{a} \xi^{b} \xi^{c}+\right.
\end{gathered}
$$




$$
\begin{gathered}
\frac{i}{8 \hbar \varepsilon} \frac{\partial^{2} g_{a b}^{\prime \prime}}{\partial \sigma^{\prime \prime c} \partial \sigma^{\prime \prime d}} \xi^{a} \xi^{b} \xi^{c} \xi^{d}+\frac{i}{8 \hbar \varepsilon} \frac{\partial g_{a m}^{\prime \prime}}{\partial \sigma^{\prime \prime c}} \frac{\partial g^{\prime \prime m n}}{\partial \sigma^{\prime \prime d}} g_{n b}^{\prime \prime} \xi^{a} \xi^{b} \xi^{c} \xi^{d} \\
\left.-\frac{1}{32 \hbar^{2} \varepsilon^{2}} \frac{\partial g_{a b}^{\prime \prime}}{\partial \sigma^{\prime \prime c}} \frac{\partial g_{d e}^{\prime \prime}}{\partial \sigma^{\prime \prime f}} \xi^{a} \xi^{b} \xi^{c} \xi^{d} \xi^{e} \xi^{f}+\ldots\right\}
\end{gathered}
$$

These expressions are replaced in Eq. (40) to obtain the infinitesimal propagator $K_{\varepsilon}\left(q^{\prime \prime} \mid q^{\prime}\right)$ entering the integration in Eq.(6). This integration is per-

formed with the help of the identities (39). Thus the wave equation results to be

$i h \frac{\partial \Psi\left(q^{\prime \prime}, t\right)}{\partial t}=-\frac{1}{2} \hbar^{2} \triangle^{(1 / 2)} \Psi\left(q^{\prime \prime}, t\right)+\frac{1}{2} \hbar^{2}\left(\left(\operatorname{det}\left(g_{a b}^{\prime \prime}\right)\right)^{\frac{1}{4}} \triangle\left(\operatorname{det}\left(g_{a b}^{\prime \prime}\right)\right)^{-\frac{1}{4}}\right) \Psi\left(q^{\prime \prime}, t\right)$

\section{ACKNOWLEDGMENTS}

This work was supported by Universidad de Buenos Aires (Proy. TX64) and Consejo Nacional de Investigaciones Científicas y Técnicas (Argentina).

\section{References}

[1] B.S.DeWitt, Rev.Mod.Phys. 29, 377 (1957).

[2] C.Morette, Phys.Rev. 81, 848 (1951).

[3] K.S.Cheng, J.Math.Phys. 13, 1723 (1972).

[4] L.Parker, Phys. Rev. D 19, 438 (1979).

[5] K.Kuchař, J.Math.Phys. 24, 2122 (1983).

[6] J.T.Whelan, Skeletonizations of phase space paths (1997), gr-qc/9709051.

[7] R.Ferraro, Phys. Rev. D 45, 1198 (1992).

[8] R.Ferraro, J. Phys. A 32, 2589 (1999).

[9] R.P.Feynman and A.R.Hibbs, Quantum Mechanics and Path Integrals, McGraw-Hill, New York (1965).

[10] J.H.Van Vleck, Proc. Natl. Acad. Sci. USA 14, 178 (1928).

[11] R.P.Feynman, Rev.Mod.Phys. 20, 367 (1948).

[12] L.S.Schulman, Techniques and Applications of Path Integration, J.Wiley, N.Y. (1981). 